\title{
Four Cases of Rhegmatogenous Retinal Detachment That Recurred More than 10 Years after Initial Reattachment by Pars Plana Vitrectomy
}

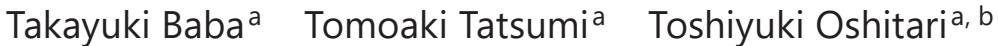 \\ Shuichi Yamamoto ${ }^{a}$ \\ aDepartment of Ophthalmology and Visual Science, Chiba University Graduate School of \\ Medicine, Chiba, Japan; ${ }^{b}$ Department of Ophthalmology, International University of Health \\ and Welfare, Narita, Japan
}

\section{Keywords}

Late recurrence $\cdot$ Rhegmatogenous retinal detachment $\cdot$ Vitrectomy

\section{Abstract}

Purpose: The purpose of this report was to present our findings in 4 cases of rhegmatogenous retinal detachment (RRD) that recurred 10,11, 12, and 17 years after a reattachment surgery by pars plana vitrectomy (PPV). Methods: Four cases of a recurrent RRD had undergone scleral buckling surgery and vitrectomy. Patients: The recurrence of the RRD was observed 10-17 years after the successful attachment by PPV. The macula was detached in all cases, and none of the eyes had severe proliferative vitreoretinopathy. The cause of the recurrence was a new retinal break in 3 eyes and a reopening of an old retinal break in the other eye. The new breaks had a punched-out shape and had neither a horseshoe tear nor an atrophic hole associated with lattice degeneration. PPV combined with scleral buckling was performed, and a reattachment was achieved in all cases. The best-corrected visual acuity (BCVA) at the last visit ranged from 20/30 to 20/25, but the BCVA in 1 eye was 20/200 because of amblyopia. Conclusions: We experienced 4 rare cases of a recurrent retinal detachment 10-17 years after the primary RRD. PPV and scleral buckling were effective and the anatomical and the functional outcomes were good. 
Baba et al.: Recurrent RRD after 10 Years Initial Attachment by PPV

\section{Introduction}

A rhegmatogenous retinal detachment (RRD) is a vision-threatening disorder, and unsuccessful treatment results in severe visual impairments. The recent advances in the treatment of RRD have resulted in a high success rates, but some cases have a persistent detachment or a recurrence of the detachment. The primary failure of attachment and an early recurrence of a detachment generally occur within 6 weeks after the primary surgery [1]. A late recurrence of a RRD (rRRD) develops 6 weeks or longer after the primary surgery. The incidence of recurrences is quite low from 0 to $4.0 \%$ at 4-6 months after a primary scleral buckle and primary pars plana vitrectomy (PPV), but it is high at $82.5-86.5 \%$ within 2 months after a primary scleral buckle and vitrectomy [2]. Lee et al. [3] reported that a rRRD is rare at 2.3\% at $\geq 6$ months after PPV. However, there is very little information on late rRRDs, especially rRRDs that develop $>10$ years after the initial surgery, and most of these cases were treated by scleral buckling $[4,5]$.

The purpose of this report was to present our findings in 4 cases of rRRD that developed between 10 and 17 years after a successful PPV for repairing a RRD. Details of the cases and postoperative courses are presented (Table 1). The patients signed an informed consent granting us permission to publish our findings. Anonymity is maintained for all patients.

\section{Case Report/Case Presentation}

\section{Case 1}

A 52-year-old Japanese woman presented with a retinal detachment at 10 years after a successful initial PPV for RRD in her left eye. The initial retinal break was observed at the superior temporal retina, and lattice degenerations were observed at the 4 quadrants. The retinal detachment involved the macula. PPV combined with cataract surgery successfully attached retina, but the visual acuity remained 20/200-20/100 due to amblyopia.

The recurrence of the RRD was noticed at 3 days before she was referred to our department for a treatment. Her best-corrected visual acuity was 20/250. The intraocular pressure (IOP) was $13 \mathrm{~mm} \mathrm{Hg}$, and the axial length (AL) was $31.56 \mathrm{~mm}$. The retina was detached at 4 quadrants and multiple breaks were present in and out of the photocoagulation scar (Fig. 1a, b). Some of the breaks were operculated tears, and some were round-shaped breaks. PPV combined with encircling SB with a 9-mm silicone tire and silicone oil endotamponade was used to reattach the retina. The silicone oil was removed, and the retina has remained attached for at least 6 years. Her visual acuity was 20/200 with an IOP of $14 \mathrm{~mm} \mathrm{Hg}$ (Fig. 1c). A domeshaped macular was observed by optical coherence tomography (Fig. 1d).

\section{Case 2}

A 70-year-old man presented with an rRRD in his left eye. The original RRD was developed 11 years earlier. There were retinal tears at the superior nasal area, and a retinal detachment was observed at the superior 2 quadrants involving the macula. He underwent phacovitrectomy, and the retina was reattached.

The retina was stable for at least 10 years. When he presented with the rRRD with a duration of 7 days, the inferior half of the retina and macula were detached (Fig. 1e, f). The former retinal breaks were sealed, and there were no other obvious breaks. His visual acuity was 20/125 with an IOP of $11 \mathrm{~mm} \mathrm{Hg}$. The AL was $24.35 \mathrm{~mm}$ in his left eye. He underwent PPV combined with a segmental buckle with a 5-mm-wide sponge over the inferior 2 quadrants. During the surgery, a small round break was observed at 4:30 o'clock. The RRD was

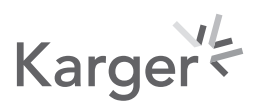


Case Reports in Ophthalmology
Case Rep Ophthalmol 2021;12:219-226

Baba et al.: Recurrent RRD after 10 Years Initial Attachment by PPV

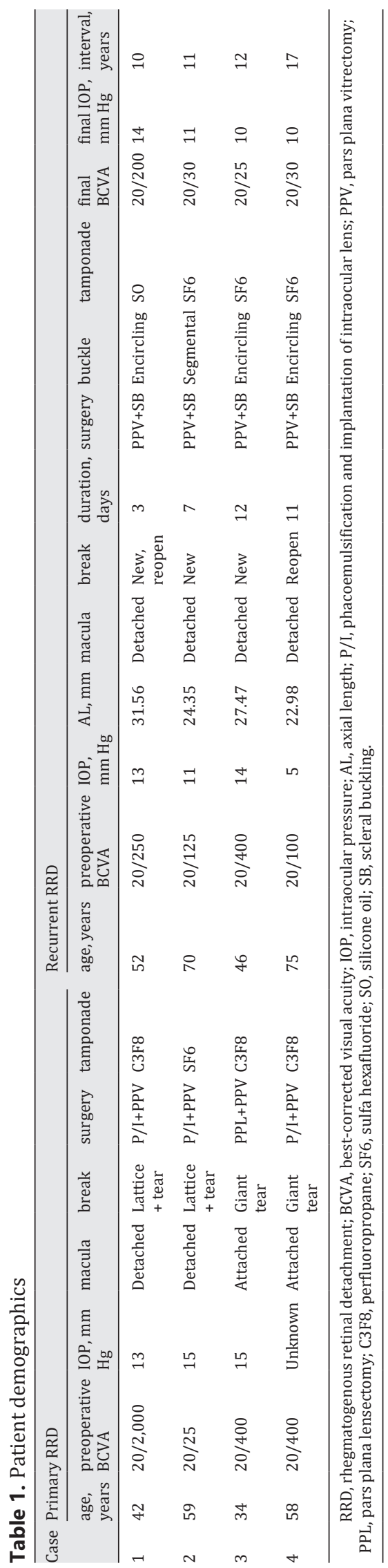


Fig. 1. Cases of a recurrent $\mathrm{RRD}$ at 10 and 11 years after a successful initial reattachment by PPV. a Fundus photograph of the left eye of a 42-yearold Japanese woman (Case 1). A retinal detachment that extended to the 4 quadrants including macula can be seen. Her visual acuity was 20/250. b Fundus drawing of the left eye showing an epiretinal membrane and reopened breaks at the superior temporal retina (arrow). There were also multiple breaks at the nasal retina (arrowhead). c Fundus photograph at 6 months after surgery. The retina is reattached, and the protrusion of the encircling scleral buckle is evident. Her visual acuity was 20/200. d OCT image showing the attached fovea with foveal depression. A dome-shaped macular can also be seen. e Fundus photograph of the left eye of a 70-year-old Japanese man (Case 2). A retinal detachment that extends to the inferior 2 quadrants including the macula can be seen. His visual acuity was 20/150. f Fundus drawing of the left eye showing an inferior retinal detachment. There is a suspicious retinal break at 6 o'clock of the retina (arrow). The scar lesions surrounding a previous break are not detached (arrowhead). g Fundus photograph at 4 months after surgery. The retina is successfully reattached with a mild protrusion of the encircling buckle. A newly treated retinal break and lattice degeneration by photocoagulation can be seen on the buckle (arrow). His visual acuity was 20/30. h An OCT image showing the attached fovea. The foveal depression is very shallow suggesting the existence of a thin epiretinal membrane. RRD, rhegmatogenous retinal detachment; PPV, pars plana vitrectomy; OCT, optical coherence tomography.
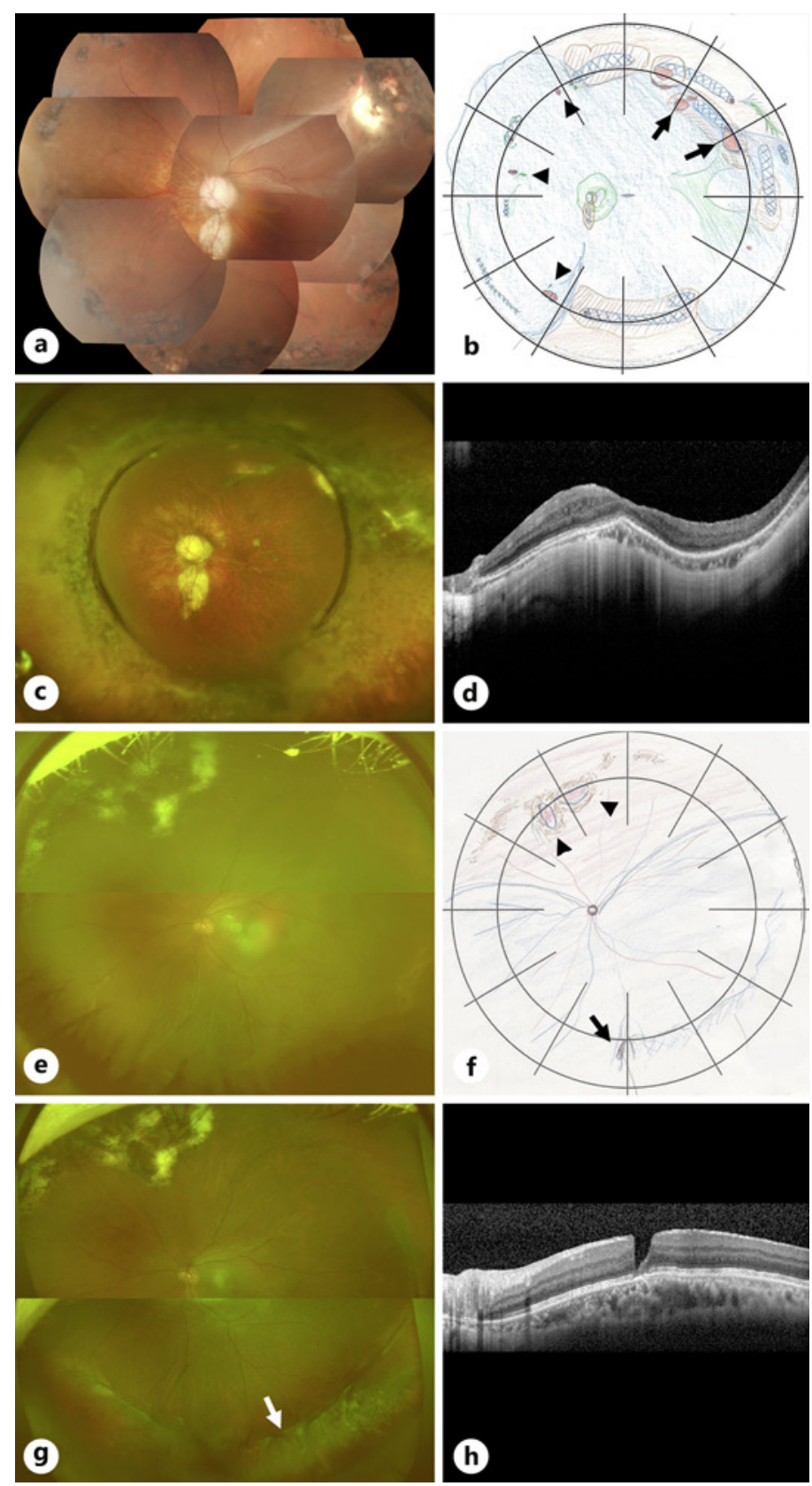

reattached by scleral buckling and PPV, and the visual acuity improved to $20 / 30$ with an IOP of $11 \mathrm{~mm} \mathrm{Hg}$ at 6 months postoperatively (Fig. 1g, h).

Case 3

A 46-year-old Japanese man presented with an rRRD. He had a history of blunt ocular trauma on his left eye at age 6 years, and he developed an RRD when he was 30 years old. The 
retinal detachment was in the superior 2 quadrants and an oral dialysis that extended about for $90^{\circ}$. He underwent PPV and lensectomy because of cataract formation. After the successful surgery, a recurrent detachment was observed when he was 34 year old. PPV and endotamponade with C3F8 were performed, and his retina was reattached.

He presented with another rRRD 12 years after the second detachment. He was aware of vision reduction 12 days earlier, and his visual acuity was 20/400, and his IOP was $14 \mathrm{~mm}$ $\mathrm{Hg}$. The left AL was $27.47 \mathrm{~mm}$. The detachment extended over 3 quadrants including the macula, and a small oval-shaped break was observed at the edge of the retinal scar at 6 o'clock (Fig. 2a, b). He underwent PPV and encircling buckle with a 7-mm silicone tire and SF6 tamponade. The retina was reattached, and his visual acuity recovered to $20 / 25$ and has been maintained for at least 19 months postoperatively (Fig. 2c, d).

Case 4

A 75-year-old Japanese man presented with a rRRD in his left eye. He had undergone phacovitrectomy for an RRD in his left eye 17 years earlier. At that time, the detachment was observed at the superior 2 quadrants, and a giant retinal tear was observed at the temporal retina. The macula was not involved. After the initial surgery, the retina was attached with a slight macular pucker.

When the rRRD developed with a duration of 11 days, his visual acuity was 20/100, and the retinal detachment involved 3 quadrants including the macula (Fig. 2e, f). The anterior part of the original break at 12 o'clock was reopened. The IOP was $5 \mathrm{~mm} \mathrm{Hg}$, and the AL was $22.98 \mathrm{~mm}$. He underwent PPV combined with an encircling buckle with a 7-mm-wide silicone tire with SF6 endotamponade. The macular pucker was removed during the surgery. The retina was successfully reattached, and his visual acuity improved to 20/30 (Fig. 2g). Optical coherence tomography showed that the epiretinal membrane was removed (Fig. $2 \mathrm{~h}$ ). His vision has been stable for 17 months.

\section{Discussion/Conclusion}

Our results showed that all 4 rRRD cases were successfully treated by PPV combined with scleral buckling surgery. We included the cases that showed the recurrence of RRD $>10$ years after the initial surgery. Such late redetachment is very rare at $2.3 \%$ at $\geq 6$ months after the initial surgery [3], and there has been no report of cases with rRRD which developed $>10$ years after the initial surgery. The cause of the rRRD has been reported to be proliferative vitreoretinopathy (PVR), a new break, and reopening of a previously treated break [6-9]. Kreissig et al. [10] reported that $5.6 \%$ of eyes had an early redetachment between 2 and 4 months, and $6.5 \%$ had a late redetachment between 3 and 7 years in their series of 107 eyes. They found the early redetachment was associated with PVR, and the late detachment was associated with new breaks. Their results are consistent with our cases which had new breaks and reopening breaks without PVR. The development of new breaks and reopening breaks suggests that a mechanism for the late recurrence is due to a residual traction by peripheral vitreous and is not due to a strong retinal traction by PVR.

Earlier studies have shown that the types of new retinal breaks observed in the rRRD [11]. These included horseshoe tears in $27.9 \%$ of the cases. On the other hand, punched-out holes, fingernail-tip-like breaks, and oval breaks which are uncommon in the primary RRD were found in the other cases. These observations were made in cases after scleral buckling and may be slightly different from our cases after PPV. There is no information about the type of break in rRRD, which developed $>10$ years after PPV, and our cases had punched-out type of breaks and had neither the typical horseshoe tears nor atrophic holes associated with

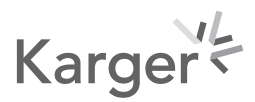




\section{Case Reports in Ophthalmology}

\begin{tabular}{l|l}
\hline Case Rep Ophthalmol 2021;12:219-226 \\
\hline DOI: 10.1159/000511372 & $\begin{array}{l}\text { ○ 2021 The Author(s). Published by S. Karger AG, Basel } \\
\text { www.karger.com/cop }\end{array}$ \\
\hline
\end{tabular}

Baba et al.: Recurrent RRD after 10 Years Initial Attachment by PPV
Fig. 2. Cases of a recurrent RRD at 12 and 17 years after the last PPV. a Fundus photograph of the left eye of a 46-year-old Japanese man (Case 3). A retinal detachment extending to the 3 quadrants including the macula can be seen. His visual acuity was 20/400. b Fundus drawing of the left eye showing a new break at 6 o'clock near the retinal scar (arrow). The other scar lesions surrounding the large breaks are not detached. c Fundus photograph at 3 months after surgery. The retina is successfully reattached, and the new break on the protrusion of the encircling buckle (arrow) is sealed. His visual acuity was 20/25. d An OCT image showing the attached fovea with foveal depression. The outer retinal structures are well restored. e Fundus photograph of the left eye of a 75-year-old Japanese man (Case 4). A retinal detachment that extends to the 3 quadrants including the macula can be seen. His visual acuity was 20/100. f Fundus drawing chart of the left eye showing a reopening of the anterior part of the break at 12 o'clock (arrow). The scar lesions surrounding a giant tear are not detached. g Fundus photograph at 3 months after surgery. The retina is successfully reattached with a mild protrusion of the encircling buckle. The opacity of the lens capsule disturbs the fundus image. His visual acuity was 20/30. h An OCT image showing the attached fovea. The foveal depression is very shallow although the epiretinal membrane had been removed during the surgery. The outer retinal structures are slightly disorganized. RRD, rhegmatogenous retinal detachment; PPV, pars plana vitrectomy; OCT, optical coherence tomography.
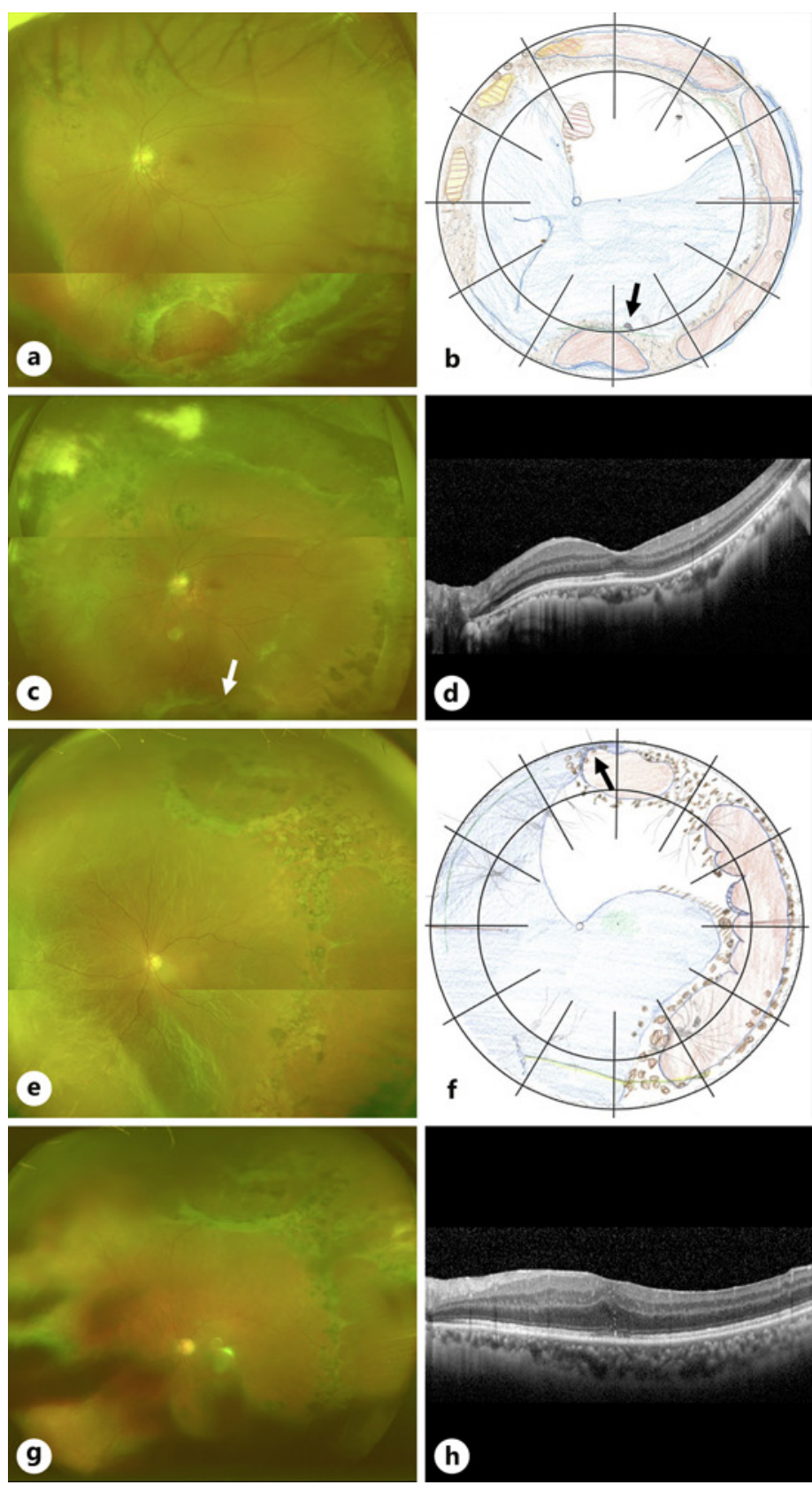

lattice degenerations. This type of break may be the typical break after PPV for RRD although we need more cases to confirm it.

The cause of the new breaks or reopening of the old breaks is believed to be vitreoretinal traction. This generally occurs with inflammation at an early postoperative period. We suggest that our cases also had vitreoretinal traction caused by a chronic contraction of residual vitreous although we could not find much vitreous cortex during the surgery. However, a 
dense, thin, and contracted vitreous remained at the peripheral retina near the vitreous base. This thin vitreous cortex was difficult to remove with a vitrectomy probe but a scleral buckle can alleviate the tension. Therefore, scleral buckling is essential for the treatment of these recurrent RRD.

A high incidence of PVR has been reported in eyes with rRRD. Rush et al. [12] reported that $76.9 \%$ of cases had PVR in their series of 104 rRRD cases. In our cases, PVR grade C or more was not observed. We suggest that our cases were examined within 2 weeks from the onset of the recurrences and had low-grade proliferation. Another possibility is that inflammation and proliferation were reduced during the many years after the initial detachment and was relatively inactive at the time of the recurrence.

In our cases, 2 eyes had high myopia with an $\mathrm{AL}>26.5 \mathrm{~mm}$. Zhioua et al. [13] reported the late rRRDs after SB (6 eyes) or PPV (3 eyes) were statistically independent of myopia. Our cases were initially treated by PPV and might have different characteristics. For example, all 4 eyes had a macula detachment which suggests a rapid development of RRD because vitreous gel was lacking.

In summary, we presented our findings in 4 cases of rRRD which developed $>10$ years after the primary reattachment by PPV. The incidence of severe PVR was low, and these cases were successfully treated by PPV with scleral buckling. The functional outcome was also favorable.

\section{Acknowledgement}

The authors thank Professor Emeritus Duco Hamasaki of Bascom Palmer Eye Institute, University of Miami, FL, USA, for providing critical discussions and suggestions to our study and editing of the final manuscript.

\section{Statement of Ethics}

The patients consented to publication of the case in writing.

\section{Conflict of Interest Statement}

The following author has no financial disclosures: T.T. and T.O. T.B.: personal fees from Bayer, personal fees from Kowa, personal fees from Santen, personal fees from Senju, personal fees from Alcon, grants and personal fees from Novartis, and outside the submitted work. S.Y.: grants and personal fees from HOYA, grants and personal fees from Senju, grants and personal fees from Pfizer, grants and personal fees from Santen, grants and personal fees from Alcon Japan, grants and personal fees from Alcon Pharma, grants and personal fees from Bayer, grants and personal fees from Kowa, personal fees from Nikon, personal fees from Wakamoto, personal fees from Chuo Sangyo, personal fees from Daiichi Sankyo, personal fees from Jamex, personal fees from Sun Contact Lens, personal fees from Finedex, personal fees from Novartis, personal fees from Chugai, personal fees from Abbvie, and outside the submitted work.

\section{Funding Sources}

This research was not supported by any competing interests of grants.

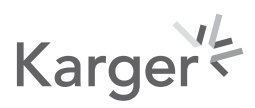


Baba et al.: Recurrent RRD after 10 Years Initial Attachment by PPV

\section{Author Contributions}

Conception and design: Baba. Data collection: Tatsumi, Baba. Analysis and interpretation: Baba, Oshitari, Yamamoto. Overall responsibility: Baba, Tatsumi, Oshirtari, and Yamamoto.

\section{References}

1 Benson W. Retinal detachment. Diagnosis and management. 2nd ed. Philadelphia, PA: J.B. Lippincott; 1988.

2 Ahmadieh H, Moradian S, Faghihi H, Parvaresh MM, Ghanbari H, Mehryar M, et al. Anatomic and visual outcomes of scleral buckling versus primary vitrectomy in pseudophakic and aphakic retinal detachment: sixmonth follow-up results of a single operation: report no. 1. Ophthalmology. 2005;112:1421-9.

3 Lee E, El Housseini Z, Steel DH, Williamson TH. An analysis of the outcomes for patients with failed primary vitrectomy for rhegmatogenous retinal detachment. Graefes Arch Clin Exp Ophthalmol. 2014;252(11): 1711-6.

4 Foster RE, Meyers SM. Recurrent retinal detachment more than 1 year after reattachment. Ophthalmology. 2002;109(10):1821-7.

5 Schmidt I, Plange N, Rößler G, Schellhase H, Koutsonas A, Walter P, et al. Long-term clinical results of vitrectomy and scleral buckling in treatment of rhegmatogenous retinal detachment. ScientificWorldJournal. 2019;2019: 5416806.

6 Michels R, Wilkinson C, Rice T. Results of retinal reattachment surgery. In: Retinal detachment. St. Louis: C.V. Mosby; 1990. p. 917-1057.

7 Schepens CL, Hartnett ME, Hirose T. Reoperations. In: Schepens's retinal detachment and allied diseases. Boston: Butterworth-Heinemann; 2000. p. 367-92.

8 Thompson JT. The repair of rhegmatogenous retinal detachments. American Academy of Ophthalmology. Ophthalmology. 1990;97(11):1562-72.

9 Nagpal M, Chaudhary P, Wachasundar S, Eltayib A, Raihan A. Management of recurrent rhegmatogenous retinal detachment. Indian J Ophthalmol. 2018;66(12):1763-71.

10 Kreissig I, Rose D, Jost B. Minimized surgery for retinal detachments with segmental buckling and nondrainage. An 11-year follow-up. Retina. 1992;12(3):224-31.

11 Menezo JL, Suarez R, Francés J. Clinical survey of the forms, number and localization of retinal tears in cases of relapses and recurrences in retinal detachment. Ophthalmologica. 1977;174(4):210-6.

12 Rush RB, Simunovic MP, Sheth S, Kratz A, Hunyor AP. Pars plana vitrectomy versus combined pars plana vitrectomy-scleral buckle for secondary repair of retinal detachment. Ophthalmic Surg Lasers Imaging Retina. 2013;44(4):374-9.

13 Zhioua R, Ammous I, Errais K, Zbiba W, Ben Younes N, Lahdhiri I, et al. Frequency, characteristics, and risk factors of late recurrence of retinal detachment. Eur J Ophthalmol. 2008;18(6):960-4. 